\title{
Precision of Navigated and Conventional Open-Wedge High Tibial Osteotomy in a Cadaver Study
}

\author{
J. Lützner, A. F. Groß, K. P. Günther, S. Kirschner \\ Department of Orthopaedic Surgery (Head: Prof. Dr. K.-P. Günther), University Hospital Carl Gustav Carus, \\ Medical Falculty of the Technical University of Dresden, Germany
}

\begin{abstract}
High tibial osteotomy (HTO) is an established treatment option for isolated medial osteoarthritis in young and active patients. One important factor for success of this procedure is the degree of correction of the weight-bearing line. Computer-assisted navigation systems are believed to improve the precision of axis correction through intraoperative real-time monitoring. This study investigates the precision of correction of the weight-bearing line in open-wedge HTO with and without a navigation system.

Nineteen legs of well-preserved human cadaver were randomly assigned to navigated ( $n=10)$ or conventional $(n=9)$ HTO. In order to achieve a sufficient amount of correction in all legs the weight-bearing line was aimed at 80 percent of the width of the tibial plateau.

The mean deviation of the weight-bearing line from the desired 80 percent was 1 percent in the navigated and 8.6 percent in the conventional operated legs $(\mathrm{p}=$ 0.002). The weight-bearing line of all navigated but only 5 of the 9 conventional operated legs was within $\mathrm{a} \pm 5$ percent tolerance level $(\mathrm{p}=0.33)$.

Navigated open-wedge HTO achieved better correction of the weight-bearing line than the conventional method in human cadaver legs. Future studies have to prove this advantage in a clinical setting and it`s effect on patient outcome.
\end{abstract}

\section{INTRODUCTION}

High tibial osteotomy (HTO) is an established treatment option in isolated unicompartmental osteoarthritis (OA) of the knee since the 1960s. [1,2] Because of technical advances in unicompartmental and total knee arthroplasty osteotomies have been performed less later on. With the implementation of new operative techniques and angle stable fixation devices this procedure currently experiences a resurgence. The goal of HTO is to transfer the load from damaged areas in the medial to the unimpaired lateral compartment, and consequently reduce symptoms and delay joint replacement. The ideal patient for an osteotomy is active and young, has a history of isolated medial compartment pain, a malalignment of less than $15^{\circ}$, a metaphyseal tibial varus, full range of motion, a BMI of less than 30 and a moderate isolated medial compartment osteoarthritis. [3]
Several studies could demonstrate that the amount of correction of the weight-bearing line is the most important factor for success. [3-8] Under- and overcorrection are the main reasons for clinical failure of this procedure. [6]

There are different methods to control the correction intraoperatively: the cable-method [9], grids [10] and the implementation of the preoperative planning. But all these methods have limitations and can lead to severe under- or overcorrection. Computer-assisted navigation systems with a system-determined error within $1^{\circ}$ [11] allow an intraoperative real-time monitoring of the leg axis and may thus improve the precision of HTO.

This study evaluates the precision of correction of the weight-bearing line in open-wedge HTO in human cadaver legs with and without the use of an imageless navigation system.

\section{Materials And Methods}

The study protocol was approved by the local independent ethics committee on 30.07.2008. From 11 human cadavers 3 legs had to be excluded due to serious restrictions in the range of motion in the hip or knee joint. 19 well preserved legs could be used for this study. These legs were randomly assigned to either navigated $(\mathrm{n}=10)$ or conventional $(\mathrm{n}=9)$ HTO. The mechanical axis and the weight-bearing line was calculated with fluoroscopic images of the hip, knee and ankle joint with the knee in full extension and neutral rotation using a calibrated grid with lead-impregnated reference lines and controlled through direct measurement on the cadaver. After HTO the weight-bearing line should pass the tibial plateau at 80 percent (medial plateau edge 0 percent, lateral plateau edge 100 percent) in order to perform a sufficient correction. A \pm $5 \%$ deviation of the weight-bearing line which is consistent with $\mathrm{a} \pm 1^{\circ}$ deviation of the mechanical leg axis was considered as acceptable tolerance level.

Preoperative planning was done graphically in all cases. The amount of correction and the size of the osteotomy gap was determined.

In all cases an imageless navigation system (OrthoPilot, Aesculap, Tuttlingen, Germany) was used to measure limb alignment before and after surgery. Data were collected with the software HTO 1.4 Open Wedge (Aesculap, Tuttlingen, Germany). Bicortical 
trackers were fixed at the distal femur und the tibia. The workflow started with palpation of the anatomic knee center with the knee $90^{\circ}$ flexed. The acquisition of the hip center was done through movement of the femur around its longitudinal axis. For registering the ankle center, a tracker was fixed at the metatarsal region using a foot plate with elastic strip, the foot being moved from extension to flexion. The knee center was located by movement of the leg from flexion to extension. Then, anatomical landmarks such as the medial and lateral epicondyle, the medial and the lateral malleolus and the anterior ankle center were registered. Then the preoperative mechanical axis in degrees varus or valgus malalignment and the intersection of the weight-bearing line with the tibial plateau was recorded.

The HTO was performed using the HTO Position spacer plate (Aesculap, Tuttlingen, Germany). An medial opening osteotomy was performed in all cases [12] and a spacer according to the preoperative planning was inserted (Fig. 1). In the navigated cases the weight-bearing line was controlled with the navigation system and the spacer was changed if necessary. In the conventional cases the correction was done according to the osteotomy gap size measured during preoperative planning. The leg alignment was controlled with fluoroscopic images of the hip, knee and ankle joint with the cable method. Then the plate was fixed with two cancellous screws proximally and two bicortical screws distally and the final anlysis of the mechanical axis and the weight-bearing line was done using the navigation system (Fig. 2) and fluoroscopic images in full extension and neutral rotation of the knee joint.

\section{STATISTICAL ANALYSIS}

Data are expressed as means with a minimum and maximum and shown graphically on box plots. Comparisons between groups were based on two sample Wilcoxon tests for continuous endpoints and on Fisher's exact tests for categorical endpoints, respectively. The results of these exploratory significance tests are summarized using $p$-values, where $p<0.05$ indicates significant differences between sub-samples. All ana- lyses were performed with the SPSS ${ }^{\circledR}$ software (SPSS Inc., Chicago, Illinois, USA, release 16.0 for Windows ${ }^{\circledR}$ ).

\section{RESULTS}

Preoperatively both groups showed a similar leg axis with a mean of $2.3^{\circ}$ of varus malalignment $\left(3^{\circ}\right.$ valgus to $7^{\circ}$ varus in the navigated group and $3^{\circ}$ valgus to $9^{\circ}$ varus in the conventional group). The weight-bearing line passed the tibial plateau at a mean of $41.5 \%$ $(17.5 \%$ to $65 \%)$ in the navigated group and at $41.4 \%$ $(9.5 \%$ to $70 \%)$ in the conventional group. There were 5 left legs in both groups.

Postoperatively the weight-bearing line passed the tibial plateau at a mean of $80.0 \%(78.0 \%$ to $81.5 \%)$ in the navigated group and at a mean of $79.4 \%(64.5 \%$ to $102.5 \%$ ) in the conventional operated group. The postoperative weight-bearing line was in all navigated cases but only 5 of the 9 conventional operated cases $(55 \%)$ within a $\pm 5 \%$ tolerance level from the desired $80 \%$ intersection of the tibial plateau $(p=0.33$, Fig. 3).

The mean deviation of the weight-bearing line from the aimed $80 \%$ intersection of the tibial plateau was $1.0 \%(0 \%$ to $2 \%)$ in the navigated and $8.6 \%(2 \%$ to $22.5 \%)$ in the conventional operated legs $(p=0.002)$.

\section{Discussion}

In cadaver [13] and clinical studies [14-16] navigated HTO was more precise in achieving the desired correction than the conventional surgical technique. In these studies radiographic or CT measurements were used as reference to evaluate the postoperative leg alignment. However, it has been shown that there is a notable inaccuracy in full-length standing radiograph to measure the leg axis due to possible flexion and rotation of the leg. [17-19] Such inaccuracies may be a major limitation for the evaluation of the mechanical axis in clinical studies. CT measurements are more precise and independent from flexion and rotation but performed in a supine position without load-bearing and may therefore not represent the physiological situ-
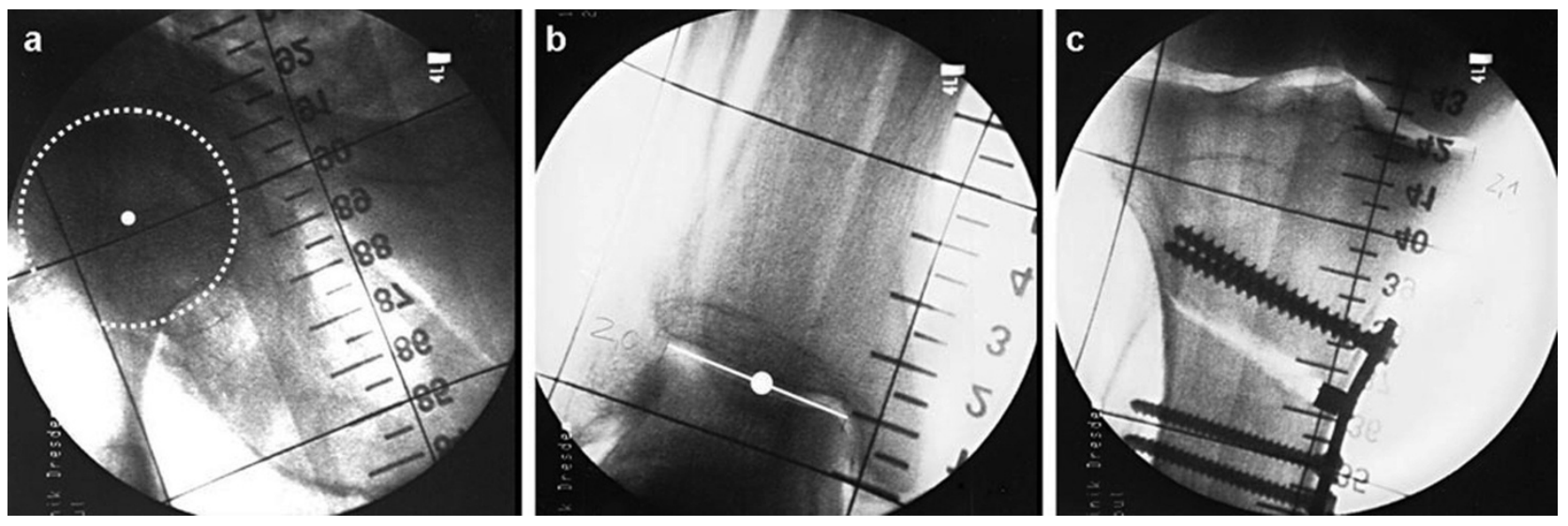

Fig. 1. Intraoperative fluoroscopic control of the leg alignment with the use of a calibrated grid with lead-impregnated reference lines: a) determination of the hip center, b) determination of the ankle center, c) open-wedge osteotomy fixed with the HTO Position spacer plate. 

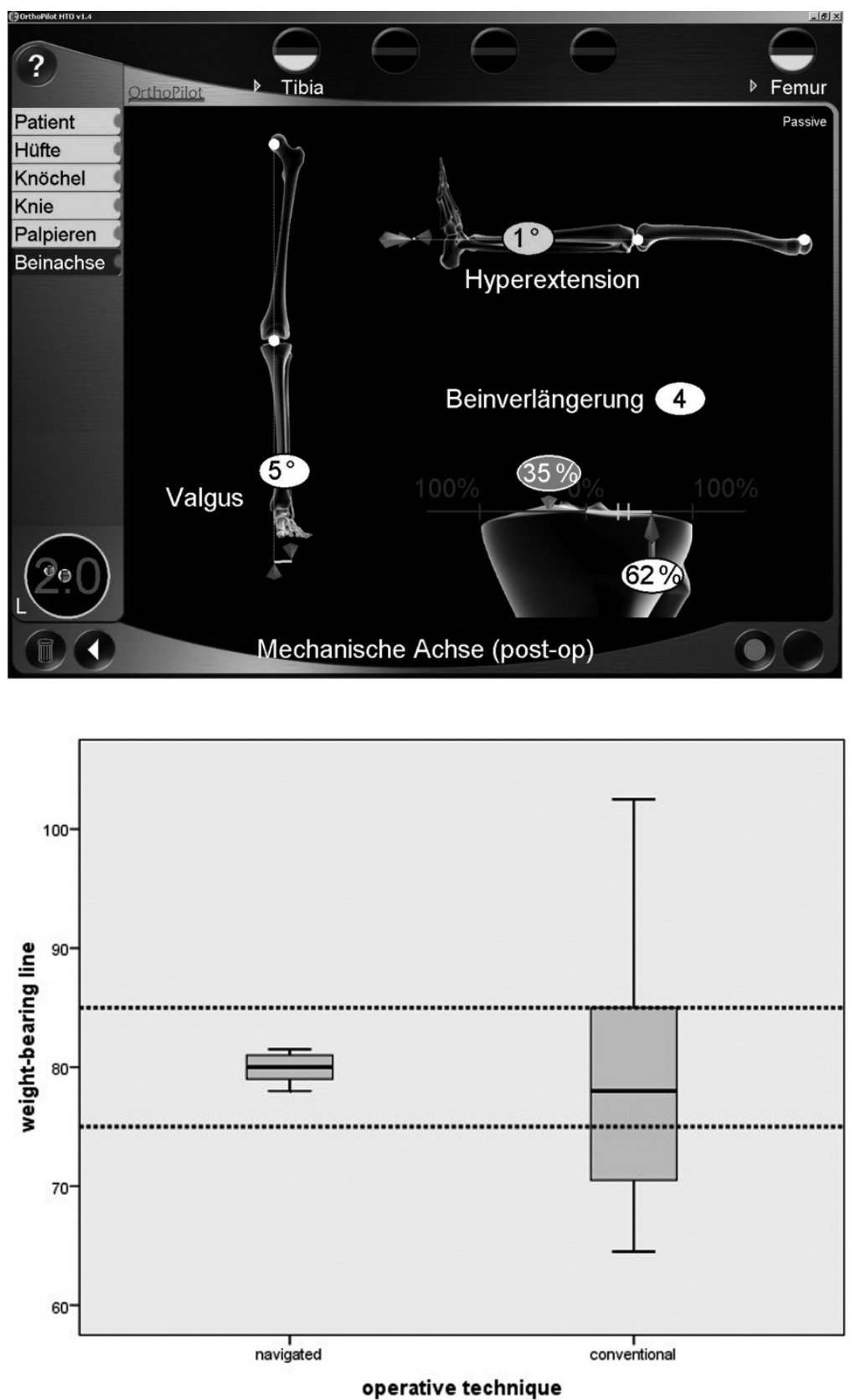

Fig. 2. Pre- and postoperative measurement of the leg alignment with the navigation system. ation. Furthermore, it has been observed that radiographic and navigation measurements of limb alignment do not correlate and it has been suggested that the navigation system may be the more precise evaluation tool. [20] It has been demonstrated that navigation systems can precisely monitor the mechanical leg axis [21-23] and that the precision is independent from the experience of the surgeon. [23] We therefore used the measurements of a navigation system as reference for evaluation of the postoperative mechanical axis and weight-bearing line. Using these measurements this study demonstrated that a defined correction of the weight-bearing line can be obtained more precise with the help of an imageless navigation system.

Limitations of this study include the experimental setting, the limited number of legs, the inclusion of legs without a varus malalignment and the measurement of the alignment only in the coronal plane and without weight-bearing. In clinical use the aim of
HTO is the intersection of the weight-bearing line at a point 30 to $40 \%$ lateral of the midpoint of the tibial plateau. [5] This is consistent with a point 65 to $70 \%$ from the medial edge of the tibial plateau. Due to legs without a varus malalignment we used a point more lateral $(80 \%$ from the medial edge of the tibial plateau) in order to perform a sufficient correction in all legs. HTO is a three-dimensional procedure and changes of the tibial slope and the rotational alignment may occur. [21, 24] We were for technical reasons not able to measure the tibial slope or the rotational alignment. We were also not able to simulate weight-bearing. This might be an additional potential of inaccuracy in patients with ligamentous instabilities.

There is no approved consensus about the necessary precision of the correction of the mechanical axis for HTO. The accepted tolerance of leg alignment of $\pm 3^{\circ}$ in total knee arthroplasty might be too large to achieve good long-term results in HTO. A tolerance 
level of $\pm 1^{\circ}$ or within $\pm 5 \%$ from the desired intersection of the weight-bearing line seems more appropriate. In this study all navigated but only $55 \%$ of the conventional operated legs were within this tolerance level. We therefore believe that the use of a navigation system can improve the clinical results of HTO. Further clinical studies are necessary to prove this.

Acknowledgements: The authors are grateful to Heike Voigt for her valuable assistance in data management and evaluation. This study has been performed within the ORTHO MIT project.

Conflict of interests statement: The authors have received no funding for this study.

\section{REFERENCES}

1. Coventry MB: Osteotomy of the Upper Portion of the Tibia for Degenerative Arthritis of the Knee. A Preliminary Report. J Bone Joint Surg Am 47:984, 1965

2. Jackson JP, Waugh W: Tibial osteotomy for osteoarthritis of the knee. J Bone Joint Surg Br 43-B:746, 1961

3. Brinkman JM, Lobenhoffer P, Agneskirchner JD, Staubli $A E$, Wymenga AB, van Heerwaarden RJ: Osteotomies around the knee: patient selection, stability of fixation and bone healing in high tibial osteotomies. J Bone Joint Surg Br 90(12):1548, 2008

4. Coventry MB, Ilstrup DM, Wallrichs SL: Proximal tibial osteotomy. A critical long-term study of eighty-seven cases. J Bone Joint Surg Am 75(2):196, 1993

5. Fujisawa $Y$, Masuhara K, Shiomi S: The effect of high tibial osteotomy on osteoarthritis of the knee. An arthroscopic study of 54 knee joints. Orthop Clin North Am 10(3):585, 1979

6. Hernigou P, Medevielle D, Debeyre J, Goutallier D: Proximal tibial osteotomy for osteoarthritis with varus deformity. A ten to thirteen-year follow-up study. J Bone Joint Surg Am 69(3):332, 1987

7. Matthews LS, Goldstein SA, Malvitz TA, Katz BP, Kaufer H: Proximal tibial osteotomy. Factors that influence the duration of satisfactory function. Clin Orthop Relat Res(229):193, 1988

8. Sprenger TR, Doerzbacher JF: Tibial osteotomy for the treatment of varus gonarthrosis. Survival and failure analysis to twenty-two years. J Bone Joint Surg Am 85A(3):469, 2003

9. Krettek C, Miclau T, Grun O, Schandelmaier P, Tscherne $\mathrm{H}$ : Intraoperative control of axes, rotation and length in femoral and tibial fractures. Technical note. Injury 29 Suppl 3:29, 1998

10. Saleh M, Harriman P, Edwards DJ: A radiological method for producing precise limb alignment. J Bone Joint Surg Br 73(3):515, 1991

11. Pitto RP, Graydon AJ, Bradley L, Malak SF, Walker CG, Anderson IA: Accuracy of a computer-assisted navigation system for total knee replacement. J Bone Joint Surg Br 88(5):601, 2006

12. Lobenhoffer P, Agneskirchner JD: Improvements in surgical technique of valgus high tibial osteotomy. Knee Surg Sports Traumatol Arthrosc 11(3):132, 2003
13. Hankemeier S, Hufner T, Wang G, Kendoff D, Zeichen J, Zheng G, Krettek C: Navigated open-wedge high tibial osteotomy: advantages and disadvantages compared to the conventional technique in a cadaver study. Knee Surg Sports Traumatol Arthrosc 14(10):917, 2006

14. Kim SJ, Koh YG, Chun YM, Kim YC, Park YS, Sung $\mathrm{CH}$ : Medial opening wedge high-tibial osteotomy using a kinematic navigation system versus a conventional method: a 1-year retrospective, comparative study. Knee Surg Sports Traumatol Arthrosc 2008

15. Maurer F, Wassmer G: High tibial osteotomy: does navigation improve results? Orthopedics 29(10 Suppl):130, 2006

16. Bae DK, Song SJ, Yoon KH: Closed-wedge high tibial osteotomy using computer-assisted surgery compared to the conventional technique. J Bone Joint Surg Br 91(9):1164, 2009

17. Krackow KA, Pepe CL, Galloway EJ: A mathematical analysis of the effect of flexion and rotation on apparent varus/valgus alignment at the knee. Orthopedics 13(8): 861,1990

18. Oberst M, Bertsch C, Wurstlin S, Holz U: [CT analysis of leg alignment after conventional vs. navigated knee prosthesis implantation. Initial results of a controlled, prospective and randomized study]. Unfallchirurg 106(11): 941, 2003

19. Ellis RE, Tso CY, Rudan JF, Harrison MM: A surgical planning and guidance system for high tibial osteotomy. Comput Aided Surg 4(5):264, 1999

20. Yaffe MA, Koo SS, Stulberg SD: Radiographic and navigation measurements of TKA limb alignment do not correlate. Clin Orthop Relat Res 466(11):2736, 2008

21. Goleski P, Warkentine B, Lo D, Gyuricza C, Kendoff D, Pearle AD: Reliability of navigated lower limb alignment in high tibial osteotomies. Am J Sports Med 36(11):2179, 2008

22. Pearle AD, Goleski P, Musahl V, Kendoff D: Reliability of image-free navigation to monitor lower-limb alignment. J Bone Joint Surg Am 91 Suppl 1:90, 2009

23. Lützner J, Gross AF, Gunther KP, Kirschner S: Reliability of limb alignment measurement for high tibial osteotomy with a navigation system. Eur J Med Res 14(10):447, 2009

24. El-Azab H, Halawa A, Anetzberger H, Imhoff AB, Hinterwimmer S: The effect of closed- and open-wedge high tibial osteotomy on tibial slope: a retrospective radiological review of 120 cases. J Bone Joint Surg Br 90(9):1193, 2008

Received: Dercember 4, 2009 / Accepted: February 17, 2010

Address for correspondence:

Jörg Lützner, MD

Department of Orthopaedic Surgery

University Hospital Carl Gustav Carus

Medical Faculty of the Technical University of Dresden

Fetscherstr. 74

01307 Dresden

Germany

Phone: +493514582613

Fax: $\quad+49351449210415$

E-mail: Joerg.Luetzner@uniklinikum-dresden.de 